\title{
Meta
}

Journal des traducteurs

Translators' Journal

\section{La recherche terminologique en traduction : pour une approche hypertextuelle}

\section{Christine Durieux}

Volume 42, numéro 4, décembre 1997

URI : https://id.erudit.org/iderudit/002687ar

DOI : https://doi.org/10.7202/002687ar

Aller au sommaire du numéro

Éditeur(s)

Les Presses de l'Université de Montréal

ISSN

0026-0452 (imprimé)

1492-1421 (numérique)

Découvrir la revue

Citer cet article

Durieux, C. (1997). La recherche terminologique en traduction : pour une approche hypertextuelle. Meta, 42(4), 677-684. https://doi.org/10.7202/002687ar
Résumé de l'article

L'auteur décrit la démarche d'un traducteur confronté à une difficulté terminologique. Constatant que la démarche mise en œuvre fait appel aux caractéristiques fondamentales d'un hypertexte, elle envisage la construction d'un hypertexte "manuel" qui pourrait servir de modèle à la construction d'un hypertexte informatisé lequel serait de nature à améliorer la performance des traducteurs tant sur le plan qualitatif que quantitatif. 


\title{
ÉTUDES TERMINOLOGIQUES ET LINGUISTIQUES
}

\section{LA RECHERCHE TERMINOLOGIQUE EN TRADUCTION : POUR UNE APPROCHE HYPERTEXTUELLE}

\begin{abstract}
Résumé
L'auteur décrit la démarche d'un traducteur confronté à une difficulté terminologique. Constatant que la démarche mise en auvre fait appel aux caractéristiques fondamentales d'un hypertexte, elle envisage la construction d" un hypertexte "manuel» qui pourrait servir de modèle à la construction d'un hypertexte informatisé lequel serait de nature à améliorer la performance des traducteurs tant sur le plan qualitatif que quantitatif.
\end{abstract}

Abstract

This article describes the process followed by a translator in a terminological research. Since this process entails resorting to the typical features inherent to hypertexts, the author suggests that a "manual» hypertext be constructed as a model for a computerized hypertext. This could enhance translators' performance both qualitatively and quantitatively.

La mise en scène de la notion même de recherche terminologique — point de départ de cette discussion - exige une brève explicitation introductive de nature à éclairer le développement ultérieur. La recherche terminologique, telle qu'elle est envisagée ici dans le cadre de l'exécution de traductions, est la recherche ponctuelle de termes, c'est-à-dire de dénominations de concepts et d'objets. Il est couramment admis que la terminologie est un «ensemble des termes qui renvoient aux concepts ou objets afférents à un domaine particulier de connaissance ou d'activité humaine» (Galisson et Coste 1976: 559). Cette définition se trouve confirmée par une autre formulation: «Ensemble d'expressions dénommant dans une langue naturelle des notions relevant d'un domaine de connaissances fortement thématisé» (Lerat 1995: 20). Ainsi donc, les termes, objets de la recherche terminologique, se trouvent-ils étroitement liés à des domaines spécialisés. À partir de là, il n'y a guère qu'un petit pas à franchir pour pouvoir apparemment affirmer que la recherche terminologique est le fait de la traduction technique. «La traduction pose des problèmes de communication, en partie cognitifs et naturels, mais aussi des problèmes linguistiques, et la traduction technique des problèmes de terminologie» (Lerat 1995: 94). Il n'est pas question ici de relancer le débat sur la relation entre langue courante et langue spécialisée, ni sur la relation entre traduction technique et traduction littéraire, qui serait tout à fait hors de propos. Il ne faudrait toutefois pas méconnaître les besoins de la traduction littéraire à cet égard. En effet, la littérature n'est pas pur style mais raconte une histoire. Cette histoire, même inventée de toutes pièces, se déroule dans un cadre qui, pour imaginaire qu'il soit, n'en est pas moins fait d'un agencement d'éléments existant par ailleurs dans le monde réel. Ainsi, la littérature décrit ou fait état de réalités, même dans un cadre de fiction, et sauf à affirmer que toute réalité est technique, force est de constater que l'exécution de la traduction d'œuvres littéraires de fiction, à l'instar de la traduction technique, exige du traducteur qu'il procède à une recherche terminologique pour découvrir ou vérifier la juste dénomination de réalités.

La recherche terminologique est donc une démarche qui s'inscrit dans le cadre de l'opération traduisante appliquée à des textes de toute nature. Elle est reliée à la traduction par un lien de subordination. Si, pour le terminologue, la traduction se situe en amont de 
la recherche terminologique qu'elle alimente en tant qu'objet d'analyse, dans cette perspective, la traduction est au service de la recherche terminologique; pour le traducteur, la relation est inverse: la recherche terminologique est un moyen de parvenir à effectuer une traduction réussie, et dans cette perspective la recherche terminologique est au service de la traduction. C'est l'optique du traducteur qui est adoptée dans le présent développement.

Pour un traducteur, la recherche terminologique se limite le plus souvent à l'utilisation en aveugle des résultats du travail du terminologue, avec la reprise pure et simple de termes proposés dans des dictionnaires ou des banques de données. Après tout, n'est-ce pas la fonction naturelle de ces outils? La remise en cause de ce réflexe simple de la part du traducteur a déjà fait l'objet d'un développement et d'une démonstration qui ont abouti à la proposition d'un arbre de décision dont le but est de s'assurer de la pertinence des correspondances figurant dans les dictionnaires bilingues pour un texte donné à traduire. De même, l'idée de fusionner recherche terminologique et recherche documentaire a déjà été présentée dans ce même ouvrage (Durieux 1988). Son application dans la pratique a largement apporté la preuve de son efficacité. Maintenant, il s'agit d'aller plus loin.

L'observation de la démarche d'un traducteur humain confronté à une difficulté terminologique a permis de retracer le chemin suivi, avec ses méandres et ses impasses, jusqu'à la découverte de la solution, avec la certitude de son adéquation au texte à traduire.

L'exemple qui va être développé relève du domaine de la construction aéronautique, mais se situe à un niveau de vulgarisation tel qu'il puisse être aisément compris par des non-spécialistes et remplir ainsi son rôle de démonstration.

Cet extrait est tiré d'un rapport sur un nouveau système de gestion de bases de données conçu à l'intention des ingénieurs et personnels techniques. Ce rapport traite longuement de la constitution d'une base de données, de son organisation, de sa gestion et des méthodes d'accès, puis donne un exemple d'application.

Engineering Applications

Wind-tunnel tests generate large quantities of data, consisting typically of measurements of:

- Overall test conditions such as windspeed, temperature and pressure, and the attitude of the model to the air stream.

- Forces and moments on the model as a whole.

- Local pressures at particular points on the surface of the model.

A small but significant proportion of tests involve measurements of a more specialised kind, such as loads on underwing slues, positions of elevators, ailerons, etc. or the velocity and direction of the flow at a number of points in the flow close to the model.

Bien entendu, conformément à nos recommandations méthodologiques habituelles, le traducteur commence par compléter ses connaissances relatives à la constitution et à la gestion de bases de données, puisque c'est le thème principal du document à traduire. À cet effet, il mène une recherche documentaire de fond. Les conditions d'exercice de la profession, et notamment les contraintes de délai, ne permettent pas au traducteur d'acquérir une somme complète de connaissances sur toutes les notions évoquées. Il est donc conduit à procéder à une recherche documentaire ponctuelle pour résoudre des difficultés thématiques ponctuelles.

L'hypothèse de départ est que le tradueteur chargé de traduire ce texte possède un bagage technique général d'un bon niveau sans être spécialisé en aéronautique ni en mécanique des fluides. Ici, la procédure proposée correspond à une démarche heuristique type ; elle est aménageable en fonction des connaissances déjà acquises par le traducteur.

Tout d'abord, il y a lieu de repérer le terme clé qui va permettre d'aborder de façon constructive le thème ponctuel. Dans le cas de cet exemple, il convient de chercher à en 
savoir plus sur wind-tunnel. À cet effet, la consultation d'un dictionnaire bilingue de l'aéronautique ouvre une porte d'entrée:

Wind-tunnel $=$ soufflerie, tunnel aérodynamique

Ce premier geste est moins la recherche d'une correspondance à adopter pour la traduction que la quête d'un point de départ pour une recherche documentaire. Déjà, pour savoir laquelle de ces deux correspondances retenir, il faut se familiariser avec l'usage dans ce domaine technique. Pour réaliser le meilleur rapport efficacité / temps passé, la consultation d'un dictionnaire encyclopédique unilingue comme le Grand Larousse fournit une définition et des explications d'un premier niveau de vulgarisation.

SOUFFLERIE - Mécanique des fluides: installation d'essai utilisée en aérodynamique pour étudier l'action sur un corps d'un écoulement d'air, de vitesse et de caractéristiques connues.

À ce stade, le traducteur est conforté; il a obtenu confirmation de ce qu'il savait confusément sur les essais en soufflerie. De plus, la définition contient le terme aérodynamique qui peut constituer une porte d'entrée en cas de besoin, puisque la seconde correspondance proposée par le dictionnaire est tunnel aérodynamique. Déjà, il est question d'écoulement d'air et de vitesse, ce qui offre une première voie de convergence textuelle, le passage étudié du texte original comportant en effet les notions de velocity and direction of the flow.

Ensuite, le texte anglais ne fait appel qu'à des notions techniques très répandues jusqu'à l'apparition de la dénomination de pièces entrant manifestement dans la construction de la maquette et, donc, dans la construction d'avions : notamment underwing slues. Ce terme est un exemple type de difficulté ponctuelle non seulement terminologique mais aussi notionnelle auquel se heurte couramment le traducteur professionnel. De nouveau, la consultation du dictionnaire bilingue spécialisé devrait lui fournir la solution avec le moindre investissement en temps et en énergie. Or, le dictionnaire bilingue de l'aéronautique ne répertorie ni underwing slue, ni underwing, ni slue. Cette notion intervenant dans une énumération, il n'y a guère que les autres membres de l'énumération pour servir de point d'appui afin d'aider à cerner la surface conceptuelle du terme. À ce stade, le traducteur sait simplement qu'il s'agit d'une pièce entrant dans la construction d'un avion. Pour en savoir plus, il lui est utile de consulter un dictionnaire unilingue anglais (Webster) pour se faire une idée de ce dont il s'agit. Là, l'entrée slue le renvoie à l'homophone slew où se trouve signalée la variante orthographique slough. Toutefois, le verbe to slue est défini, pour un contexte de navigation maritime, et donne une idée de virage.

Ainsi donc, d'après la motivation du terme underwing, le traducteur comprend que les underwing slues sont des pièces qui se situeraient sous l'aile d'un avion et qui en outre serviraient à virer. Par ailleurs, l'extrait du rapport indique bien que, dans la soufflerie, le flux d'air exerce sur ces pièces des charges qu'il y a lieu de mesurer. Il s'agit donc de pièces situées de façon à opposer une résistance à l'écoulement de l'air. À l'issue de ce raisonnement, le point focal de la recherche documentaire devient une aile d'avion. Là encore, la démarche la plus économique en temps est sans doute la consultation d'un dictionnaire encyclopédique (Larousse). Á l'entrée aile, dans la rubrique relative à l'aéronautique, on peut lire :

AÉRONAUTIQUE - surface horizontale sur laquelle s'exercent les forces aérodynamiques de portance assurant la sustentation d'un avion.

[...] La face supérieure de l'aile s'appelle extrados et la face inférieure intrados. Le bord antérieur se nomme bord d'attaque, le bord postérieur bord de fuite, et les extrémités latérales bords marginaux. Une aile est caractérisée géométriquement par sa vue en plan [...] 
Sur l'aile sont disposés les volets et les ailerons nécessaires à la manœuvre de l'appareil, et aussi en général les freins aérodynamiques.

Ce développement s'accompagne d'une illustration rudimentaire, avec quelques légendes, mais pas les désignations intrados, extrados, volet. Nous constatons que l'acquisition pas à pas de connaissances thématiques permet de se former progressivement une image mentale de l'objet désigné. Toutefois, cette image est encore imprécise; mais de nouveaux termes clés sont apparus, qui vont servir de pivots pour la construction de notre hypertexte «manuel»: intrados qui, déjà, semble pouvoir correspondre à underwing et volets et/ou ailerons pourraient désigner les pièces dénommées slues. D'après la formation du terme underwing, on peut raisonnablement déduire que ce doit bien être la face inférieure de l'aile qui est ainsi désignée. Toutefois, il peut être utile d'en savoir plus sur l'emploi du terme intrados. Une rapide consultation du dictionnaire encyclopédique (Larousse) à l'entrée intrados confirme les connaissances acquises jusqu'à présent.

AÉRONAUTIQUE - Surface inférieure d'une aile. La surface opposée, qui constitue le dessus de l'aile, se nomme extrados.

Une encyclopédie, telle l'Encyclopædia Universalis, peut fournir des explications plus approfondies que celles que donne un simple dictionnaire encyclopédique, mais l'investissement en temps que requiert sa consultation est plus lourd, c'est la raison qui nous fait préférer d'abord la consultation la plus économique. L'entrée dans l'encyclopédie exige d'abord la consultation du thesaurus : aile d'avion envoie à l'article aérodynamique (vol. 1 : 302-331). Cet article s'articule en cinq chapitres. Le premier - Considérations théoriques - comporte de très nombreuses formules mathématiques d'un niveau technique trop élevé et non pertinent pour le traducteur. Le deuxième - Viscosité : concept de couche limite - également riche en formules mathématiques, est encore extérieur à notre sujet. Le troisième - Applications - comporte des illustrations qui retiennent l'attention: il y est manifestement question d'écoulement de l'air par rapport à des profils d'aile. Ce chapitre est long (10 pages) mais sa lecture rapide va permettre de repérer des informations pertinentes:
3. Applications
Avions subsoniques
[...] des volets hypersustentateurs augmentent la courbure du profil de l'aile, et par conséquent la portance; on augmente encore l'efficacité de ces volets en ménageant entre le volet et l'aile une fente qui permet aux filets d'air de passer de l'intrados à l'extrados, réalisant un effet de soufflage qui retarde le décollement. (p. 310)

Les explications sont d'un niveau très spécialisé, ce qui exclut la présence d'une simple illustration morphologique d'une aile d'avion. Toutefois, le traducteur se sent en confiance, familiarisé avec l'emploi d'un terme nouveau pour lui, l'intrados, qu'il retrouve une nouvelle fois; de même, il retrouve le terme volet qui va lui fournir un nouveau point de départ pour consulter, de façon rapide, le dictionnaire encyclopédique (Larousse). Là enfin, il va trouver la solution à son problème.

VOLET - Aéronautique: volet d'intrados, volet hypersustentateur situé sous la partie inférieure de l'aile, au niveau du bord de fuite, et pouvant s'escamoter en vol normal dans l'aile.

En procédant de la sorte, le traducteur a réalisé une sorte d'hypertexte manuel (cf. figure 1). Pour finalement acquérir la certitude qu'il y a lieu, dans ce texte, de traduire underwing slues par volets d'intrados, il lui aura fallu réaliser neuf manipulations. 
(1) Consultation du dictionnaire bilingue de l'aéronautique Wind-tunnel $=$ soufflerie, tunnel aérodynamique

(2) Consultation du dictionnaire encyclopédique Larousse - Soufflerie Mécanique des fluides : installation d'essai utilisée en aérodynamique pour étudier l'action sur un corps d'un écoulement d'air, de vitesse et de caractéristiques connues.

(3) Consultation du dictionnaire bilingue de l'aéronautique Underwing slue, non répertorié Slue, non répertorié

(4) Consultation du dictionnaire unilingue anglais Webster Slue $\rightarrow$ Slew $\rightarrow$ Slough $=$ idée de virage

(5) Consultation du dictionnaire encyclopédique Larousse - Aile Aéronautique: surface horizontale sur laquelle s'exerce les forces aérodynamiques de portance assurant la sustentation d'un avion.

... La face supérieure de l'aile s'appelle extrados et la face inférieure intrados. Le bord antérieur se nomme bord d'attaque, le bord postérieur bord de fuite, et les extrémités latérales bords marginaux. Une aile est caractérisée géométriquement par sa vue en plan ... Sur l'aile sont disposés les volets et les ailerons nécessaires à la manœuvre de l'appareil, et aussi en général les freins aérodynamiques.

(6) Consultation du dictionnaire encyclopédique Larousse - Intrados Aéronautique : surface inférieure d'une aile. La surface opposée, qui constitue le dessus de l'aile, se nomme extrados.

(7) Consultation du Thesaurus de l'Encyclopaedia Universalis Aile d'avion $\rightarrow$ Aérodynamique

(8) Consultation de l'Encyclopaedia Universalis - Aérodynamique 3. Applications Avions subsoniques ... des volets hypersustentateurs augmentent la courbure du profil de l'aile, et par conséquent la portance; on augmente encore l'efficacité de ces volets en ménageant entre le volet et l'aile une fente qui permet aux filets d'air de passer de l'intrados à l'extrados, réalisant un effet de soufflage qui retarde le décollement.

(9) Consultation du dictionnaire encyclopédique Larousse - Volet Aéronautique: volet d'intrados, volet hypersustentateur situé sous la partie inférieure de l'aile, au niveau du bord de fuite, et pouvant s'escamoter en vol normal dans l'aile.

Il est effectivement permis de parler ici d'hypertexte, car la démarche mise en œuvre fait bien appel aux caractéristiques fondamentales d'un hypertexte (Balpe 1990). De fait, (1) les dictionnaires et les encyclopédies consultées constituent un ensemble d'informations textuelles au sein duquel les parcours de lecture (cf. figure 2) peuvent être multiples. (2) Le multifenêtrage est présent avec la possibilité de garder ouverts, à la page voulue, plusieurs volumes en même temps et de se reporter de l'un à l'autre à volonté. (3) L'interactivité est assurée puisque c'est le lecteur qui choisit son parcours de lecture à tout moment en fonction de sa progression vers la solution finale.

En outre, le modèle hypertextuel ainsi construit répond aux six principes qui caractérisent l'hypertexte (Lévy 1990).

(1) Le principe de métamorphose: le réseau hypertextuel est sans cesse en construction et en renégociation. À chaque instant, le lecteur effectue des choix de nature à orienter sa lecture pour la faire progresser vers l'objectif visé. Selon son niveau de connaissances préalablement acquises, le traducteur peut choisir de chercher à en savoir plus sur le fonctionnement d'une soufflerie ou d'un tunnel aérodynamique, par exemple, ou encore de se reporter à un ou à plusieurs des corrélats pertinents indiqués à la fin de l'article Aérodynamique de l'Encyclopædia Universalis tels que Fluides (Mécanique des) ou encore Vol (Mécanique du). 
(2) Le principe d'hétérogénéité: les nœuds et les liens d'un réseau hypertextuel sont hétérogènes. Il peut s'agir de textes descriptifs, explicatifs, définitoires, ou autres; il peut aussi s'agir d'images. Ici, par exemple, le traducteur recherche des correspondances de termes mais aussi la description de la morphologie d'une aile d'avion, l'explication du fonctionnement des volets, la définition de l'intrados et encore une illustration d'une aile d'avion. De plus, dans les liens, la logique sous-jacente peut revêtir différentes formes: reprise d'un mot clé (5-7), avec réduction (3-4), avec effet de zoom (5-6), correspondances terminologiques (0-1), association d'idées (3-5), confirmation (2-8); les liens logiques peuvent aussi relever de l'antonymie, l'analogie, l'isotopie, etc.

(3) Le principe de multiplicité et d'emboîtement des échelles : l'hypertexte s'organise en mode fractal, c'est-à-dire que n'importe quel nœud ou n'importe quel lien peut lui-même se révéler composé de tout un réseau, et cela indéfiniment. Ainsi, dans la recherche terminologique, l'effet de zoom consistant à rechercher la définition d'un terme contenu dans la définition d'un autre terme peut se perpétuer indéfiniment. Ce principe est également appelé isomorphisme.

(4) Le principe d'extériorité : le réseau ne possède pas d'unité organique, sa construction dépend à tout moment de l'ajout d'éléments nouveaux venus de l'extérieur. Le nombre d'ouvrages consultables est infini. Les éléments d'information sont constamment puisés dans des sources extérieures au réseau qu'ils viennent étoffer par incrémentation.

(5) Le principe de topologie: le réseau hypertextuel se construit et fonctionne de proche en proche. Il n'y a pas de cadre bornant un espace imposé. L'hypertexte se développe librement en empruntant non pas des chemins préexistants, mais les chemins qu'il crée lui-même en se construisant.

(6) Le principe de mobilité des centres : le réseau hypertextuel n'a pas de centre ou s'il se trouve qu'à un moment donné il en ait un, celui-ci se trouvera vite déplacé ou remplacé l'instant d'après, puisque la construction du réseau évolue en permanence et que ce dernier présente donc une géométrie variable.

La métaphore de l'hypertexte reste au niveau de l'analogie. Il y a analogie mais non coïncidence entre le fonctionnement de l'ordinateur et celui de l'esprit humain. D'abord, l'un et l'autre présentent une architecture très différente. Si ni l'un ni l'autre ne sont capables de fournir des solutions à partir de rien, le capital de départ du cerveau humain est déjà présent à la naissance, même s'il s'étoffe au fil de la vie et de l'expérience. L'ordinateur, lui, n'a «à la naissance» que de la matière inerte; il exécute ensuite des opérations en fonction des instructions et des données qui lui ont été fournies, et il faut bien qu'elles lui aient été fournies, sans elles, il ne peut rien faire. La supériorité du cerveau humain se manifeste aussi dans son organisation hiérarchisée et son aptitude à effectuer de nombreuses activités parallèles simultanément. Par ailleurs,

[...] le langage de l'homme et celui de l'ordinateur sont fondamentalement différents. L'homme complète, précise ses paroles par des gestes, des expressions. Il utilise l'exagération, le sous-entendu, des figures de rhétorique. Il emploie des mots à sens multiples [...] La machine traite des caractères, des formes, et ne peut pas avoir accès au sens des mots; chaque symbole ou groupe de symboles doit toujours avoir le même sens. (Lazorthes $1988: 80)$

Néanmoins, l'intelligence artificielle cherche à singer l'intelligence humaine en reprenant son architecture, en imitant son mode de raisonnement logique, en se dotant d'une capacité de traitement parallèle neuronal et en s'efforçant de fonctionner en langage naturel. En conséquence, il est envisageable de créer des outils d'hypernavigation dans des ressources documentaires permettant une lecture de type hypertextuel comparable à celle que le traducteur humain met en cuvre pour mener à bien une recherche termino- 
logique ponctuelle. En tant que reflet des besoins et de la démarche naturelle de l'esprit humain, cet hypertexte «manuel» pourrait servir de modèle à la construction d'un hypertexte informatisé. La construction, sur support informatique, de réseaux tels que celui que nous venons de décrire serait de nature à améliorer la performance des traducteurs tant sur le plan qualitatif - qualité des traductions produites - que sur le plan quantitatif - délai plus bref d'exécution des traductions.

CHRISTINE DURIEUX Université de Caen, France

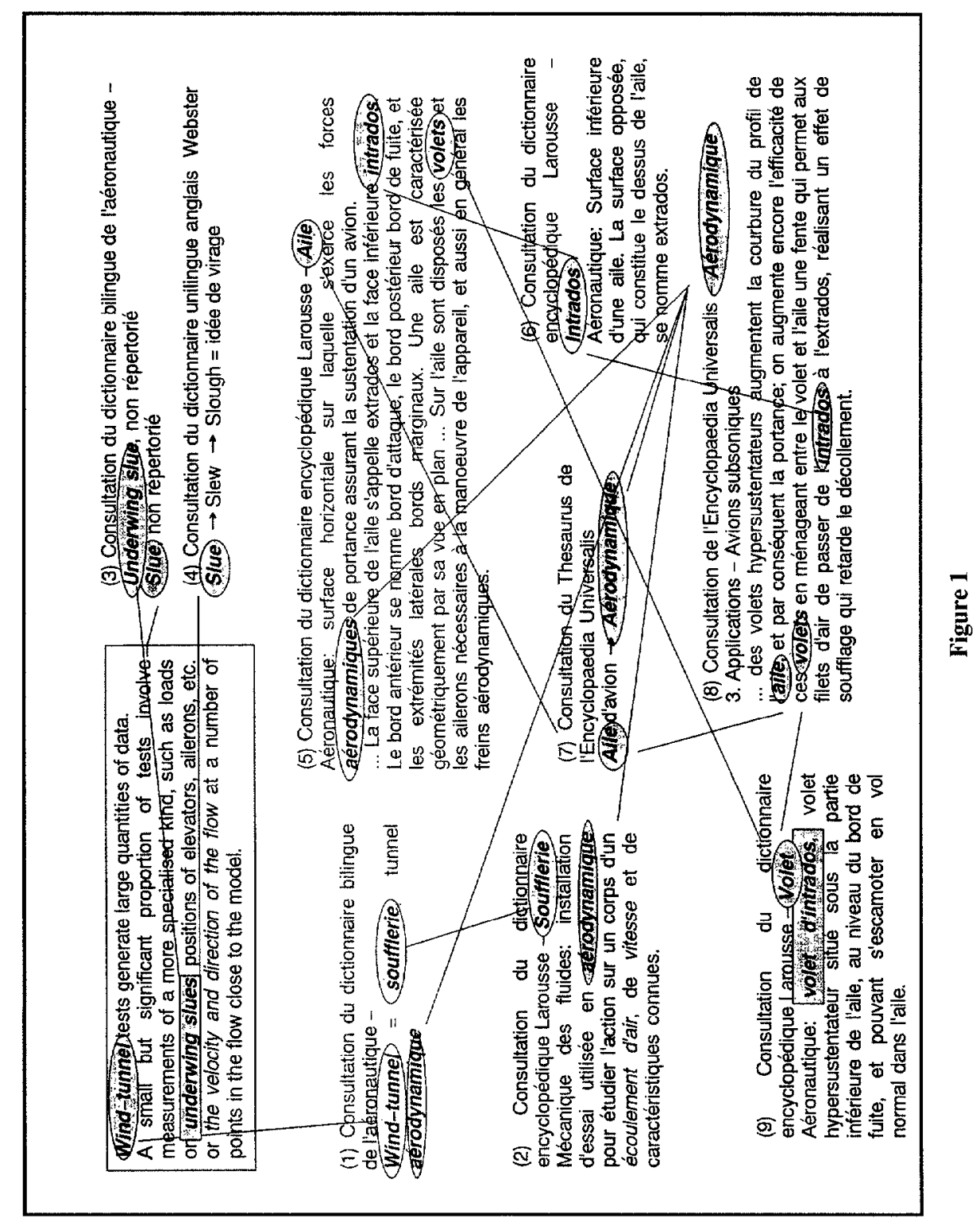




\section{PARCOURS DE LECTURE}

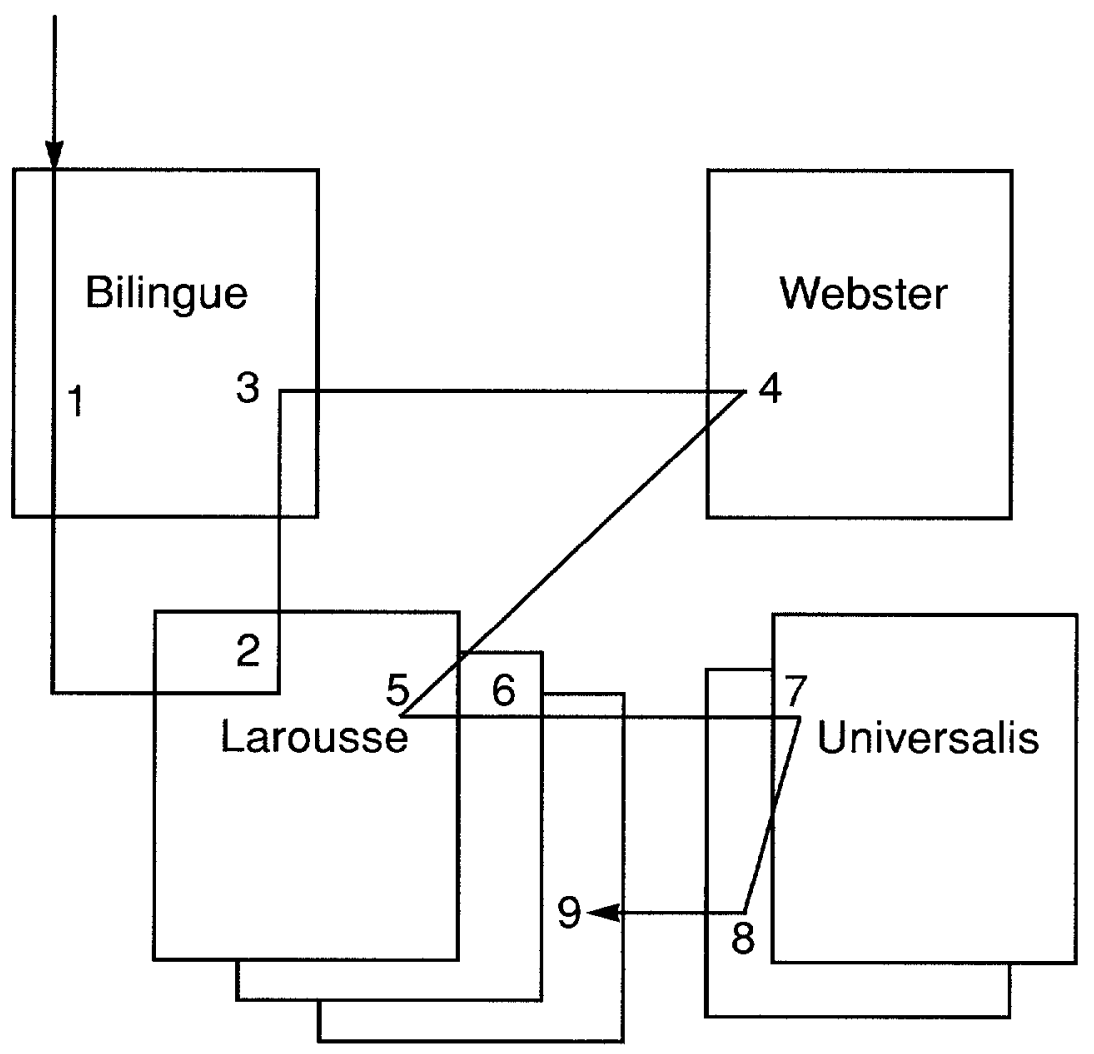

Figure 2

\section{RÉFÉRENCES}

BALPE, Jean-Pierre (1990): Hyperdocuments, Hypertextes, Hypermédias, Paris, Eyrolles.

BUSH, Vannevar (1945): «As We May Think», Atlantic Monthly.

DURIEUX, Christine (1988): Fondement didactique de la traduction technique, Paris, Didier Érudition.

DURIEUX, Christine (1994) : «Texte, Contexte, Hypertexte», Cahier du CIEL, Université Paris VII, pp. 214-228.

GALISSON, Robert et Daniel COSTE (1976): Dictionnaire de didactique des langues, Paris, Hachette.

LAZORTHES, Guy (1988): Le cerveau et l'ordinateur, Toulouse, Privat.

LERAT, Pierre (1995): Les langues de spécialité, coll. «Linguistique nouvelle», Paris, PUF, 206 p.

LÉVY, Pierre (1990): Les technologies de l'intelligence. L'avenir de la pensée à l'ère informatique, Paris,

La Découverte.

MINSKY, Marvin (1986) : The Saciety of Mind, New York, Simon \& Schuster. 